\title{
EVALUACIÓN INTEGRAL DE LA SEGURIDAD ESTRUCTURAL DE EDIFICACIONES EXISTENTES DAÑADAS POR SISMOS DE GRAN MAGNITUD
}

\author{
${\text { Daniela Giovanna Razo } \text { Carrasco }^{(1)} \text { y Octavio García Domínguez }}^{(1)}$
}

\begin{abstract}
RESUMEN
Se presenta una metodología para realizar una evaluación integral de la seguridad estructural de edificaciones de concreto reforzado existentes, afectadas por sismos intensos con base en trabajos de campo y resultados de análisis numéricos. También puede ser aplicada a construcciones nuevas sobre las cuales se tengan incertidumbres de su integridad o comportamiento estructural, o bien a estructuras que han sido reforzadas y se desea verificar la eficacia de este. La metodología incluye recomendaciones para el monitoreo del deterioro de la rigidez lateral y la integridad estructural, a través de la evolución de los parámetros que caracterizan el comportamiento dinámico estructural.
\end{abstract}

Palabras clave: seguridad estructural; inspección ocular; daños por sismo; vibración ambiental; calibración estructural

\section{COMPREHENSIVE ASSESSMENT OF THE STRUCTURAL SAFETY OF EXISTING BUILDINGS DAMAGED BY LARGE-MAGNITUDE EARTHQUAKES}

\begin{abstract}
A methodology for comprehensive assessment of the structural safety of existing reinforced concrete buildings affected by intense earthquakes, based on field works and numerical analysis results, is presented. It can also be applied to new constructions on which there are uncertainties of their integrity or structural behavior, or to structures that have been reinforced and it is desired to verify its effectiveness. The methodology includes recommendations for lateral stiffness deterioration and structural integrity monitoring, through the evolution of the parameters that characterize the structural dynamic behavior.
\end{abstract}

Keywords: structural safety; visual inspection; seismic damage; environmental vibration; structural calibration

\footnotetext{
Artículo sobre el tema central de la tesis ganadora del Primer Concurso Nacional de Tesis de Licenciatura sobre Temas de Ingeniería Sísmica 2019 organizado por la SMIS. Se aceptarán comentarios y/o discusiones hasta cinco meses después de su publicación.

(1) Departamento de Estructuras DICyG, Facultad de Ingeniería, Universidad Nacional Autónoma de México, Ciudad Universitaria, CDMX, C.P. 04510.giovannarazo96@gmail.com,posgradoingcivil@ingenieria.unam.mx
} 


\section{INTRODUCCIÓN}

La verificación de la seguridad estructural de las construcciones es una práctica de vital importancia para evitar pérdidas humanas y económicas, producto de las acciones estáticas y/o dinámicas a las que pueden estar sujetas durante su vida útil. Dadas las condiciones de sismicidad que prevalecen en gran parte de nuestro país, el diseño de las edificaciones regularmente está regido por las acciones sísmicas y sus efectos sobre éstas, constituyendo una de las áreas de estudio más importantes en el campo de la ingeniería estructural. En esta área trabajan distintos grupos de académicos, investigadores, empresas y profesionales de la práctica, cuyas contribuciones han permitido mejorar y desarrollar nuevas normas de diseño para contar con construcciones más seguras. Actualmente, los reglamentos de diseño sísmico evalúan la seguridad estructural de las construcciones mediante el cumplimiento de estados límite de servicio y de falla, como es el caso del RCDF (GCDMX, 2017), el cual se aplica en la zona metropolitana de la Ciudad de México y se usa como referencia en distintas entidades federativas de nuestro país. De acuerdo con este código, se alcanza un estado límite de comportamiento en una construcción cuando se presenta una combinación de fuerzas, desplazamientos, niveles de fatiga, o varios de ellos, que determina el inicio o la ocurrencia de un modo de comportamiento inaceptable de dicha construcción.

La inobservancia del estado límite de servicio puede generar efectos perceptibles capaces de alterar o perturbar la funcionalidad de una edificación y/o el nivel de confort de los usuarios. Entre los efectos más comunes se encuentran las vibraciones y deformaciones excesivas en la estructura, que no permitan el correcto uso de las instalaciones o que requieran la modificación del inmueble. Por otro lado, el incumplimiento del estado límite de falla, también llamado estado límite de resistencia, pone en riesgo la capacidad y estabilidad de la estructura ante acciones de diseño. La excedencia de ambos estados límite se manifiesta a través de diversas evidencias físicas y/o analíticas que pueden ser detectadas mediante diferentes niveles de evaluación. De acuerdo con el artículo 179 del reglamento actual y sus NTC (GCDMX, 2017), las estructuras del grupo B que no presentan daño, no están obligadas a reforzarse; sin embargo, las estructuras del grupo A deben cumplir con la capacidad sismorresistente derivada de la actualización de las normas de diseño.

\section{ANTECEDENTES}

\section{Efectos de los sismos en las edificaciones}

Los efectos que generan los eventos sísmicos en las edificaciones son variables y dependen de diversos factores entre los que se pueden mencionar: la edificación (material, estructura, antigüedad, etc.), el sismo (origen, tipo, intensidad, etc.) y el terreno sobre el cual esta cimentada la edificación. Es sabido que, dependiendo de la localización de la fuente sísmica y de las características del suelo, entre otros factores, las frecuencias con las que se transmiten los diferentes trenes de ondas sísmicas pueden generar movimientos de la masa de suelo que causan devastación a grupos específicos de estructuras. Por ejemplo, según los datos históricos y levantamientos posteriores al sismo del 19 de septiembre de 1985, la mayoría de las estructuras que se dañaron o colapsaron fueron edificios altos, con periodos de vibración mayores a 1 seg. y el daño se concentró en la zona centro de la Ciudad de México, es decir, suelo arcilloso de origen lacustre con periodos dominantes de vibración del suelo mayores a $1 \mathrm{~s}$.

Fue a partir de este evento sísmico, cuando se evidenciaron en México los daños que puede ocasionar el fenómeno de resonancia, el cual es provocado por la coincidencia o cercanía de frecuencias de vibración, asociadas a los tres factores antes mencionados, es decir, la frecuencia de vibración de la estructura, la frecuencia de la onda sísmica y la frecuencia natural del suelo. El efecto de la resonancia sobre las 
estructuras se traduce en una amplificación de las fuerzas sísmicas causando graves daños y colapsos parciales y/o totales en las edificaciones, como se muestra en la fig. 1.

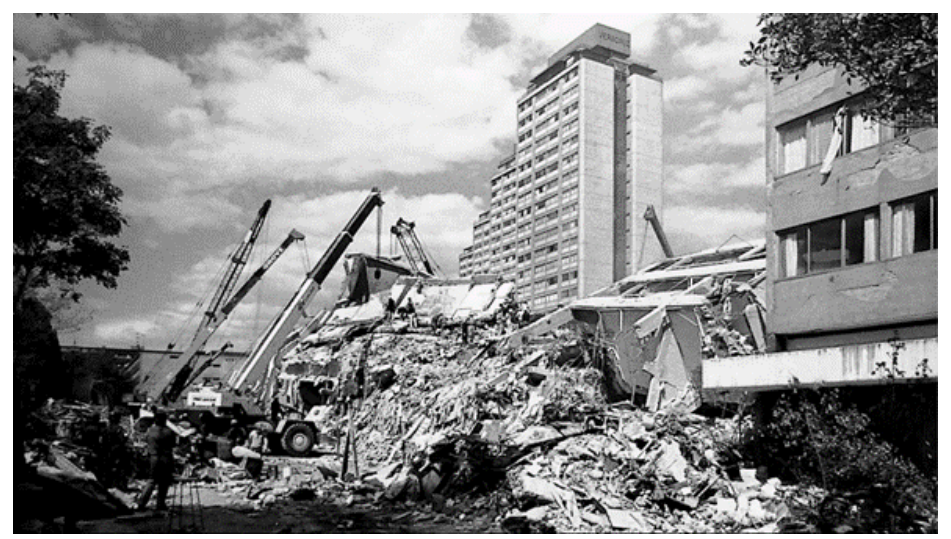

Figura 1. Derrumbe del edificio Nuevo León, 1985 en la Ciudad de México. (Protocolo, 2015)

A diferencia del sismo de 1985, por las características del sismo del 19 de septiembre del 2017, los daños se concentraron en las estructuras de baja a mediana altura; la mayoría de los derrumbes se localizaron en la zona de transición y la zona de lago de Ciudad de México, figs. 2 y 3.; y en los estados de Morelos y Puebla, donde los daños se presentaron principalmente en iglesias, mercados, edificios gubernamentales y viviendas; en algunos municipios las afectaciones alcanzaron hasta el $90 \%$ de las edificaciones.

Además de los aspectos antes mencionados, existen factores de vulnerabilidad sísmica asociados al tipo de estructuración, a su configuración arquitectónica e incluso a su uso y ubicación en la manzana; algunos de estos, son la irregularidad en planta y elevación, el entrepiso débil (también conocido como planta baja flexible), losa plana, efectos de columna corta, impacto de estructuras en colindancia, masas excéntricas e irregulares, esbeltez, longitud excesiva en planta, entre otros. Los factores de irregularidad han causado comportamientos sísmicos no deseables que han sido identificados durante diversos sismos en el mundo. A pesar de lo anterior, los reglamentos aún permiten estos diseños y configuraciones, por lo que es común encontrarlos en estructuras con todo tipo de usos como oficinas, hospitales, casa habitación, etc.

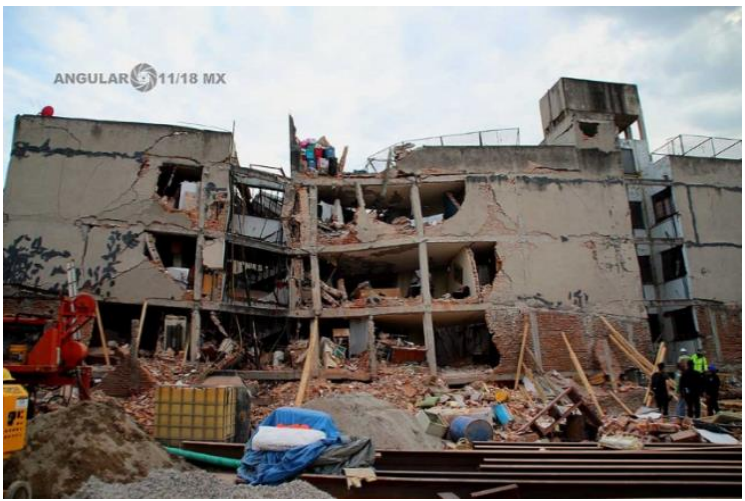

Figura 2. Daño severo y derrumbe parcial del edificio de 5 niveles en Saratoga 715, Colonia Portales, Ciudad de México. Vista lateral. (ANGULAR 11-18 MX, 2017)

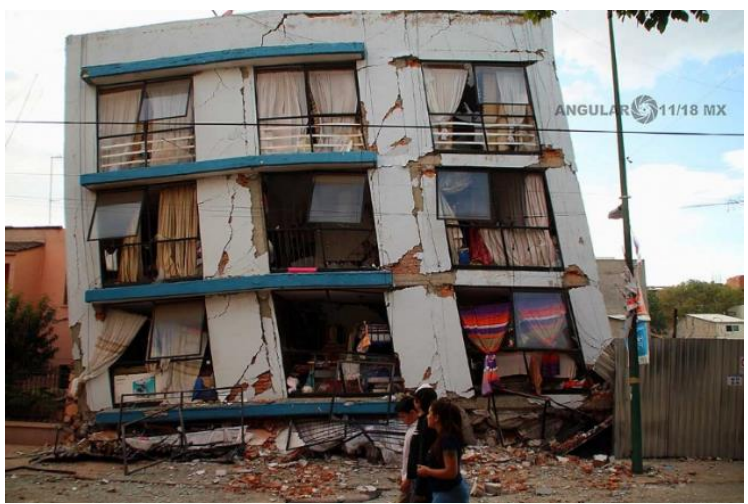

Figura 3. Daño severo y derrumbe parcial del edificio de 5 niveles en Saratoga 715, Colonia Portales, Ciudad de México. Vista frontal. (ANGULAR 11-18 MX, 2017) 


\section{Normatividad vigente}

En México, cada entidad federativa es libre de redactar sus propios reglamentos con el fin de adaptarlos a sus condiciones. En caso de no contar con uno, generalmente se hace referencia al RCDF (GCDMX, 2017), así como al MDOC (CFE, 2015). Ambos documentos cuentan con normas específicas para el diseño y construcción de estructuras. Es de importancia mencionar que, en la última actualización de la reglamentación, se incorporaron capítulos correspondientes a Evaluación y Rehabilitación a las NTCDCEC (GCDMX, 2017) y las NTC-DCEM (GCDMX, 2017), lo cual, constituye un avance importante en materia de evaluación y rehabilitación de estructuras dañadas.

Los requisitos básicos de la seguridad estructural de las edificaciones son tratados en el cuerpo principal del RCDF, la definición y determinación de estados límite son descritos en las NTC (GCDMX, 2017). Además de dichos manuales y reglamentos, el CENAPRED cuenta con manuales de evaluación postsísmica y de rehabilitación de estructuras que son usados en todo el país.

\section{La seguridad estructural de las edificaciones}

Debido a los graves efectos que pueden derivarse de los fenómenos naturales como los sismos, se torna de mayor importancia el considerar correctamente las demandas sísmicas que tienen mayor posibilidad de ocurrir en una determinada región; sin embargo, es imposible garantizar que una estructura podrá permanecer en pie o resistir todos los sismos que ocurran durante su vida útil, sin daños importantes. Por ello, las autoridades de cada país se encargan de elaborar reglamentos y normas en busca de establecer límites o estándares mínimos de seguridad adaptados a cada zona geográfica. Debido a lo anterior, la seguridad de una construcción es una condición estructural que se encuentra asociada al cumplimiento de estados límite, para un nivel de demanda sísmica establecido en un reglamento específico.

Según la filosofía y criterios para la protección civil establecidos en los manuales de revisión postsísmica, también se deberá considerar la posible ocurrencia de un accidente derivado del colapso de elementos no estructurales o contenidos en el interior de la edificación, por ello, no debe confundirse el concepto de seguridad estructural, con la seguridad de los ocupantes, ya que la primera está asociada a la estabilidad de la edificación y, la segunda, al riesgo que representa ingresar o permanecer en el inmueble.

\section{METODOLOGÍA PARA LA EVALUACIÓN INTEGRAL DE LA SEGURIDAD ESTRUCTURAL DE LAS EDIFICACIONES}

\section{Proceso de evaluación y metodología}

El proceso de evaluación debe fungir como un filtro, el cual podrá escalar a un nivel superior, en función de los resultados de la primera valoración; por ejemplo, si durante una evaluación rápida de emergencia realizada por personal de Protección Civil, no se encontraron daños o peligros para los usuarios, no será necesaria realizar una inspección ocular detallada por un ingeniero civil, ni mucho menos se realizará una evaluación integral de la seguridad estructural por especialistas. Los niveles de evaluación varían según los protocolos de cada país y región. En la fig. 4, se describen los 3 niveles que comúnmente se realizan en México. 

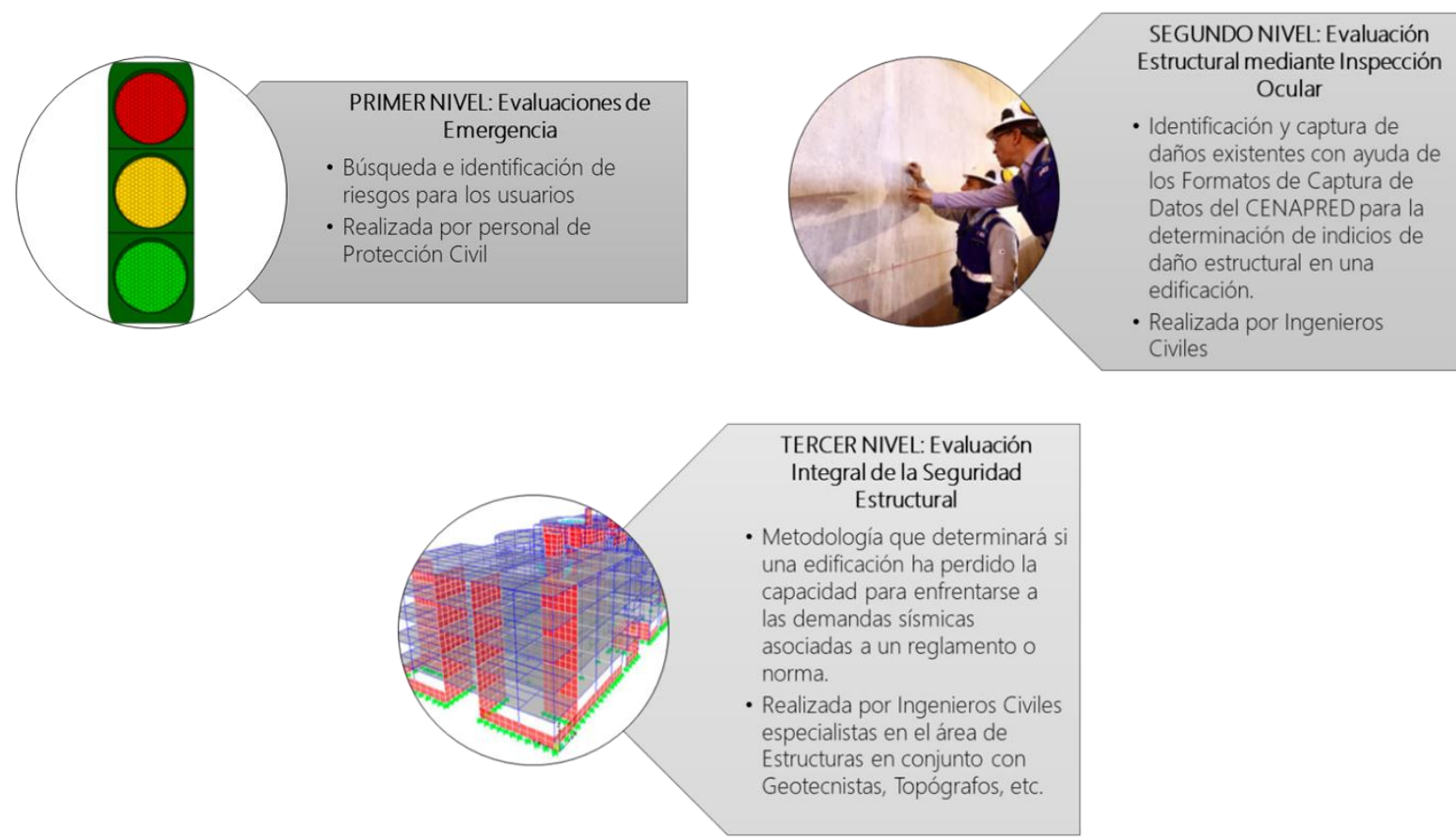

Figura 4. Niveles de evaluación estructural

Una vez que se ha diagnosticado el posible daño estructural, el tercer nivel, identificado como la evaluación integral de la seguridad estructural, será la herramienta para determinar la integridad y estabilidad del inmueble acorde a los requisitos establecidos en la normatividad vigente en la zona. Estas evaluaciones proporcionan el nivel más alto de confiabilidad para dictaminar las condiciones estructurales de una edificación y, con base en ello, proponer las medidas necesarias para mejorar su potencial sísmico. La metodología de evaluación estará conformada por diferentes etapas, tanto en gabinete como en campo, que tendrán como resultado el desarrollo de un modelo estructural de la edificación en condiciones actuales, con el cual se podrá revisar el cumplimiento de los estados límite establecidos por la reglamentación. En la fig. 5, se presenta un diagrama de flujo que ilustra las etapas de esta metodología. 


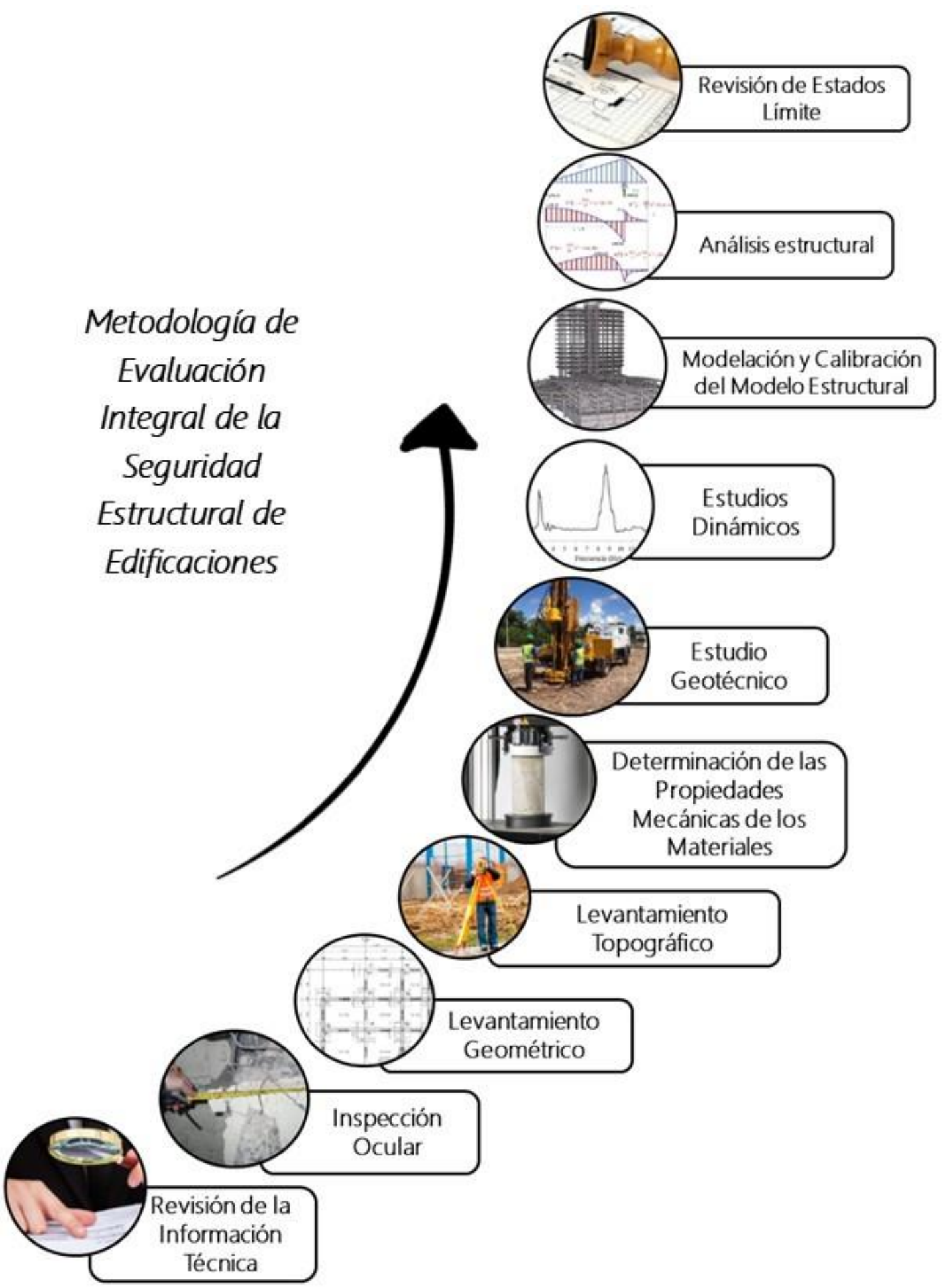

Figura 5. Metodología de evaluación de la seguridad estructural de las edificaciones 


\section{Revisión de la información técnica}

La existencia de planos arquitectónicos, memoria de cálculo, planos estructurales, estudios previos (levantamientos topográficos, sondeos geotécnicos, etc.), así como modificaciones, ampliaciones o reforzamientos a lo largo de la vida útil de la estructura, serán elementos útiles para la definición de los alcances de la evaluación, así como para la planeación de los trabajos de campo y de gabinete requeridos para el objetivo buscado, fig. 6.

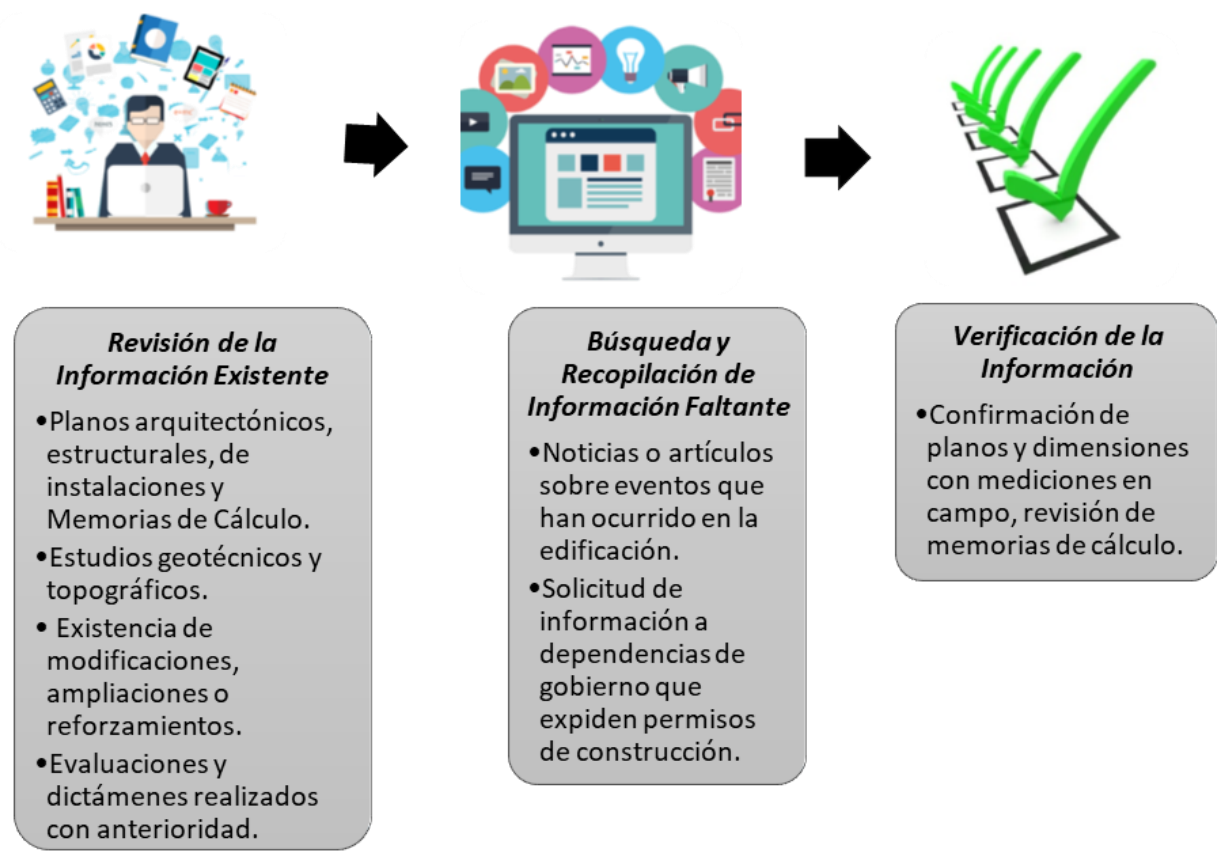

Figura 6. Aspectos que deben considerarse en la revisión de la información técnica

La existencia de evaluaciones y/o dictámenes estructurales derivados de la ocurrencia de eventos sísmicos pasados, proporcionará información sobre el estado de la estructura antes del daño que se busca diagnosticar. En algunos casos podría ser factible identificar aspectos como acumulación de daño, disminución de la rigidez lateral en la estructura o un deterioro de los materiales, ayudando a dar un diagnóstico mucho más completo.

En muchos casos, la información existente será nula, inexacta e inclusive errónea, por lo que será de suma importancia la verificación de toda la información mediante pruebas directas o indirectas y levantamientos estructurales propios, que permitan identificar posibles cambios durante la construcción o modificaciones posteriores que afectarían las características reales de la estructura.

Con base en lo antes expuesto, la definición, los alcances y planeación de los trabajos de gabinete, de campo y de laboratorio, deberá realizarse después de una visita de exploración en la cual se realice un levantamiento rápido de las condiciones del inmueble, de manera que dichas actividades se puedan realizar de la forma más eficiente y segura.

\section{Inspección estructural ocular detallada}

La inspección estará enfocada en el reconocimiento visual no destructivo de todas las partes asequibles de la estructura, con objeto de identificar el estado actual de integridad de sus materiales y 
elementos estructurales, que permita hacer un diagnóstico general del comportamiento de la estructura en función del tipo y características del daño encontrado; con esta información se podrán proponer algunos estudios y trabajos posteriores para realizar una evaluación estructural más detallada, como la que se describe en este trabajo.

La inspección ocular ayudará a identificar evidencias de mecanismos patógenos de comportamiento que pudieran existir o estar presentándose en la estructura, los cuales deberán documentarse detalladamente con ayuda de reportes fotográficos o videos. Para realizar estos trabajos, es necesario definir una metodología, criterios y formatos de inspección, que serán utilizados por el personal encargado de realizar esta tarea. Se recomienda formar brigadas que realicen recorridos reportando daños estructurales y, de ser el caso, daños no estructurales que pongan en riesgo a los ocupantes. Entre los daños más comunes en estructuras de concreto se pueden encontrar:

- Fisuras, grietas y/o fracturas producto de efectos de cortante y flexión

- Deterioro de materiales por agentes ambientales (Humedad, salitre, fuego, etc.)

- Pérdida de recubrimiento

- Aplastamiento del concreto

- Exposición y/o corrosión del acero de refuerzo

- Carbonatación

- Pandeo local del acero de refuerzo longitudinal

- El daño inducido por el paso inadecuado de instalaciones

- Flechas o deformaciones excesivas de trabes, losas y voladizos

- Efectos de punzonamiento en losas planas y aligeradas

- Efectos de cortante por columna corta, etc.

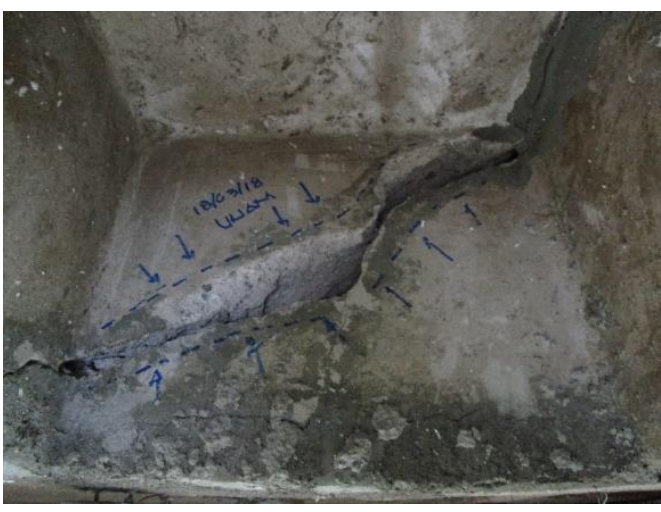

Figura 7. Fractura en trabe. (DE-DICYG, 2018)

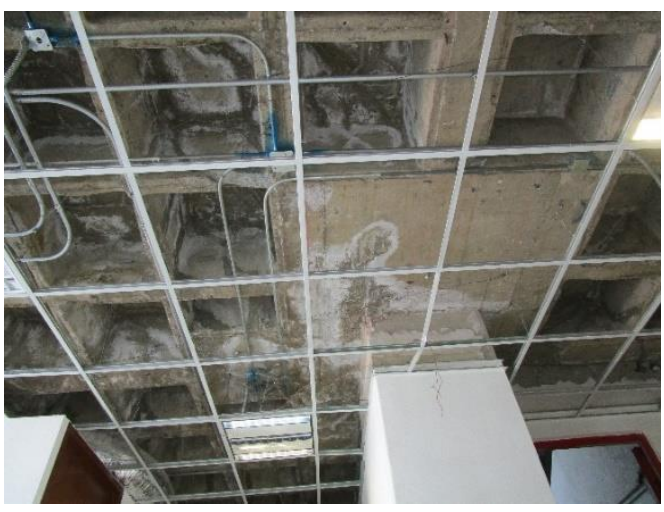

Figura 8. Humedad en concreto. (DE-DICYG, 2018) 


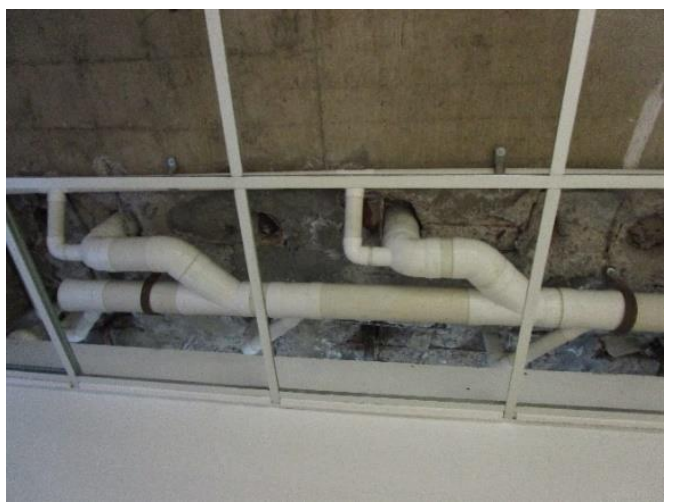

Figura 9. Paso inadecuado de instalaciones. (DE-DICYG, 2018)

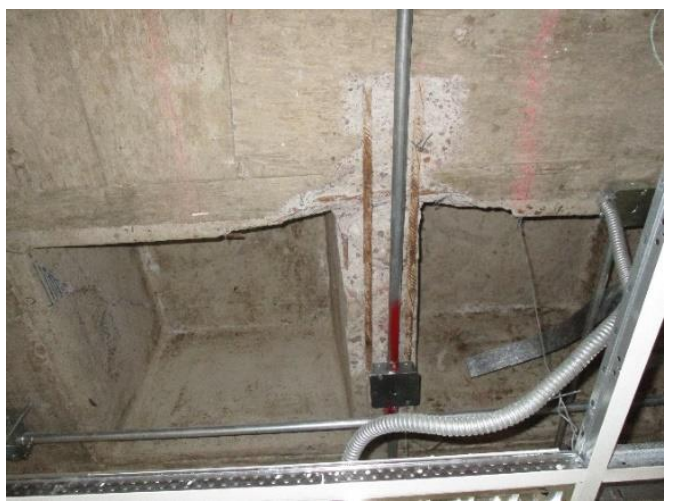

Figura 11. Pérdida de recubrimiento. (DE-DICYG, 2018)

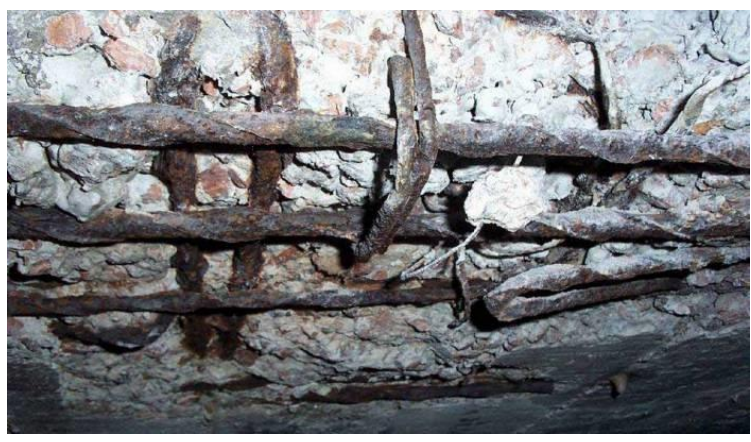

Figura 13. Carbonatación y grave deterioro del concreto. (Materiales Ramonsón, 2013)

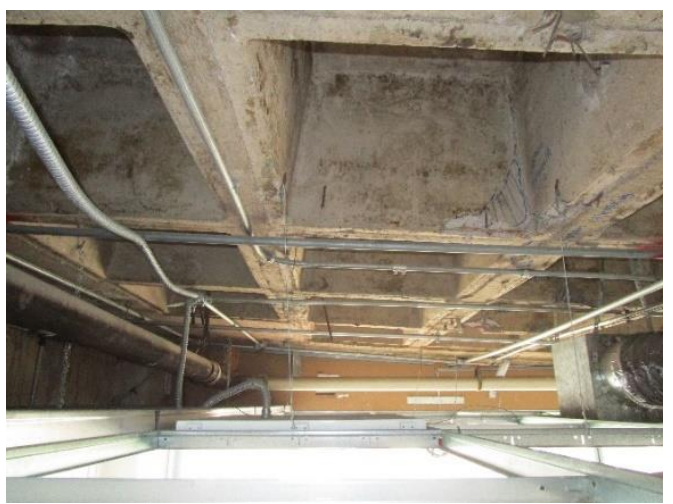

Figura 10. Flecha en trabes. (DE-DICYG, 2018)

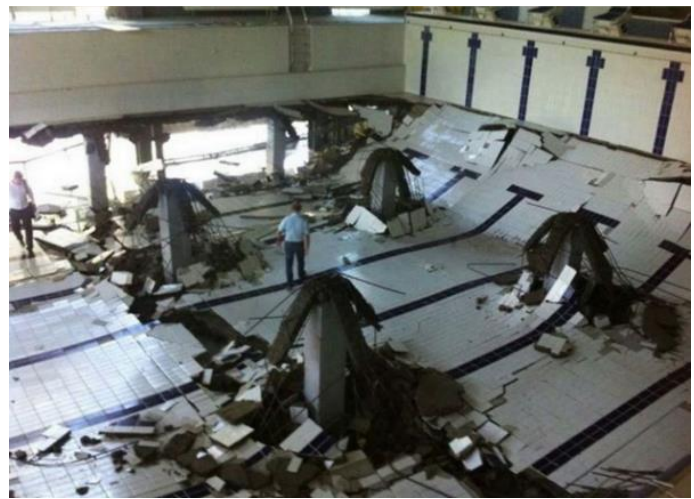

Figura 12. Efectos de punzonamiento (blogs.20minutos, 2016)

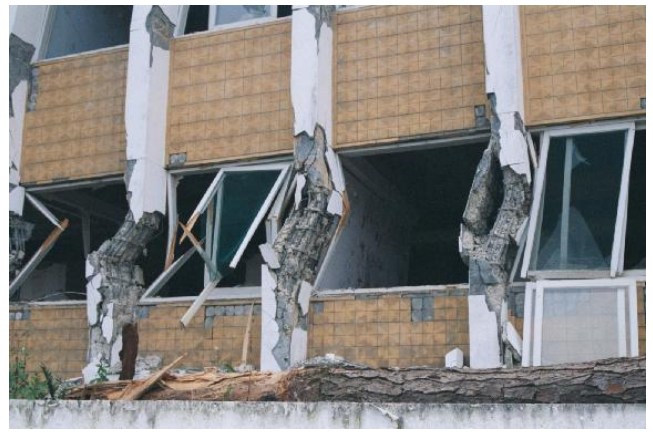

Figura 14. Efectos de columna corta. (@Mundo_Hormigon, 2017)

A partir de los resultados de un estudio topográfico, es posible identificar y cuantificar el nivel de asentamientos diferenciales o desplomos, que pueden generar daños importantes en una construcción. La inspección de la cimentación y documentación de daños comúnmente se realiza por parte de un especialista en geotecnia, quién a su vez analiza los resultados derivados de la exploración del subsuelo y realiza la evaluación geotécnica. La correcta interpretación del comportamiento de la cimentación podrá ayudar a identificar zonas potenciales de daño en la superestructura, por ello la importancia de recabar todo tipo de 
evidencias en campo. En las figs. 15-18. se muestran algunos ejemplos de los efectos causados por fallas en suelo o cimentación.

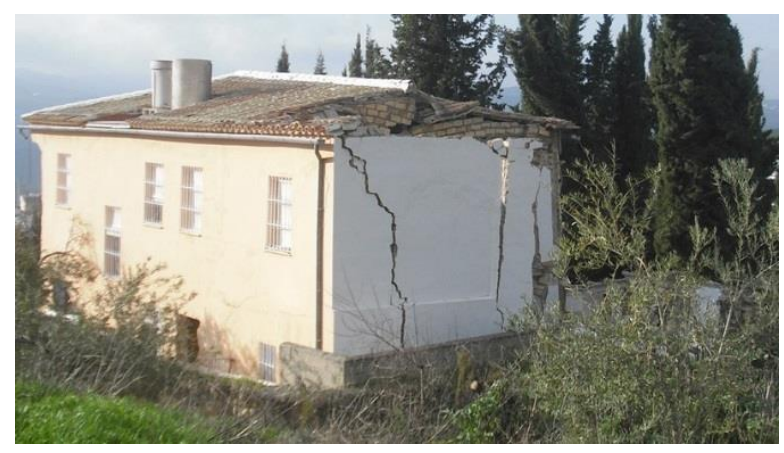

Figura 15. Efectos por asentamientos diferenciales. (geotecniafacil.com, 2017)

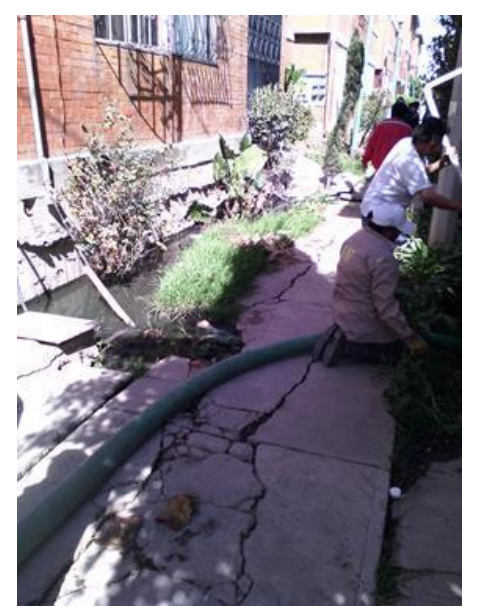

Figura 17. Asentamientos en terrenos circundantes de la construcción. (DE-DICYG, 2018)

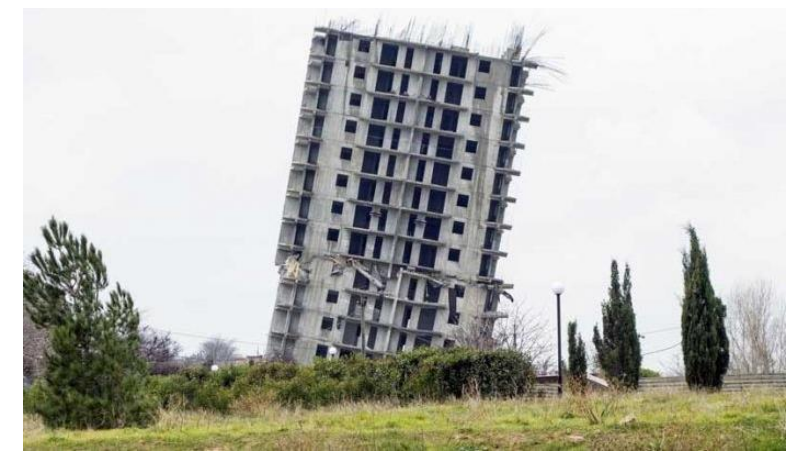

Figura 16. Pérdida de verticalidad por falla del suelo. (IDC, 2019)

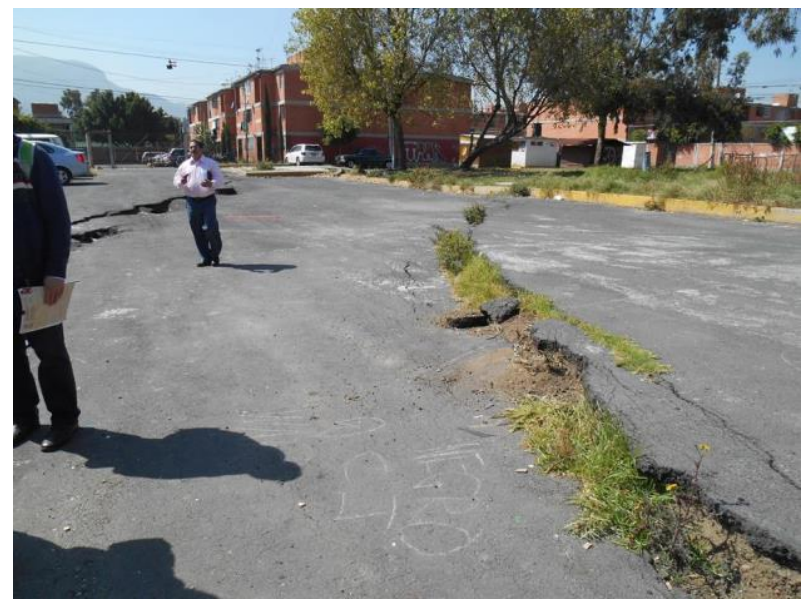

Figura 18. Fallas regionales. (DE-DICYG, 2018)

Con objeto de evitar pérdidas en la información recabada se recomienda preparar formatos de inspección dependiendo del sistema estructural existente. Para casos de edificaciones grandes la logística será primordial. Las tareas de inspección y levantamiento de daños deben realizarse poniendo atención en los siguientes aspectos:

- La correcta orientación y revisión de las caras del elemento estructural

- La medición del largo y espesor de las grietas, así como de cualquier deterioro que se presente en el elemento.

- La adecuada señalización y clasificación de los patrones de agrietamiento en los elementos estructurales con ayuda de marcadores, en los que se indique el grosor de la grieta y la fecha de medición. Una adecuada señalización de daños permitirá, entre otras cosas, monitorear su posible evolución.

- La documentación completa del estado físico del elemento con ayuda de fotografías de los daños existentes, así como de la inexistencia de estos. 
En caso necesario, se realizará el retiro de los acabados o recubrimientos de los elementos estructurales con el fin de observar con la mayor claridad posible la profundidad del daño.

Dependiendo de las condiciones de la edificación, el equipo necesario para la inspección puede variar; sin embargo, el material básico debe incluir lo siguiente:

- Herramientas para remover o demoler acabados y recubrimientos (Cincel, martillo, desarmadores, etc.)

- Escaleras y linternas

- Instrumentos de medición como los grietómetros, nivel, flexómetros, cintas, plomadas, etc.

Será obligatorio el uso de equipo de seguridad básico: casco, chaleco, lentes de protección y cubre bocas. Se recomienda el uso de tablas para escribir, croquis y planos, plumones, marcadores y lápices para llevar un control de las áreas revisadas. Se sugiere realizar bitácoras y programas de avance, ya que, en estructuras de dimensiones mayores, estas tareas pueden durar varias semanas.

\section{Pruebas para determinar propiedades mecánicas de materiales}

La realización de pruebas para determinar las características y propiedades mecánicas de los materiales es una de las etapas más importantes para contar con la información que permita modelar la estructura en sus condiciones actuales. Factores como la edad de la construcción, el daño acumulado y agentes ambientales o químicos, afectan la integridad del concreto y del acero. Por ello, a pesar de contar con información sobre las características de los materiales especificados en el proyecto original, siempre debe realizarse un programa completo de pruebas y ensayes que confirmen o ajusten dichos valores.

Las pruebas que se pueden realizar en los elementos para la determinación de propiedades suelen clasificarse en destructivas y no destructivas. Las primeras consisten en la extracción de probetas o demolición de elementos que posteriormente requerirán reparaciones; y las segundas correlacionan datos obtenidos de manera indirecta para dar una aproximación de las propiedades.

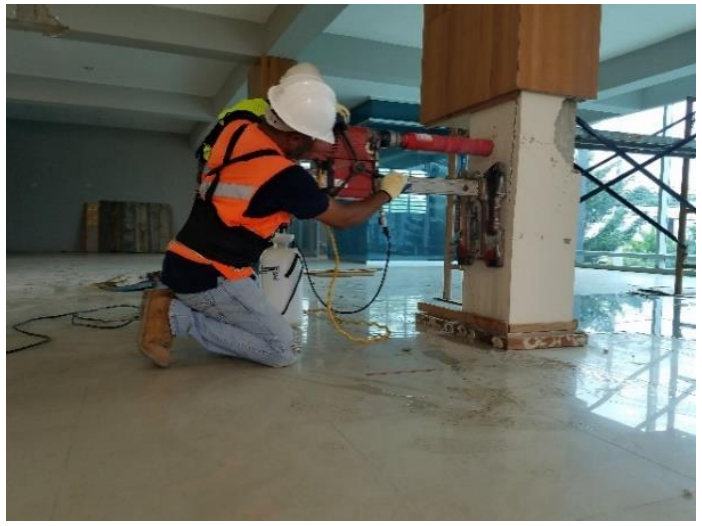

Figura 19. Extracción de núcleo de concreto. (INDECAL, 2019)

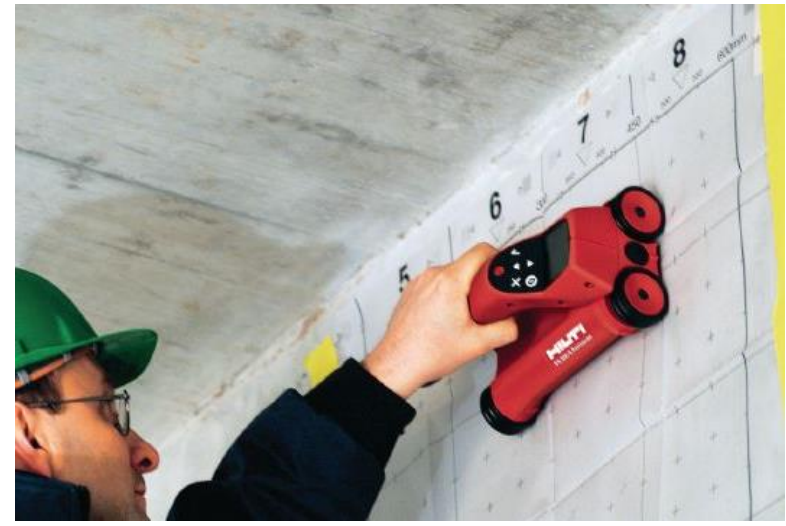

Figura 20. Utilización del dispositivo FerroScan en campo. (Hilti México, 2019)

\section{Levantamiento geométrico}

La realización de esta etapa dependerá de la calidad de la información existente del proyecto original del inmueble ya que, si después de una verificación rápida en sitio, se determina que los planos estructurales 
existentes son representativos de la estructura, no será necesario realizar un levantamiento geométrico detallado. Es recomendable, identificar si se realizaron cambios en la estructura durante la construcción que no se ven reflejados en los planos estructurales, así como los reforzamientos o ampliaciones que pudo sufrir la edificación, fig. 21.

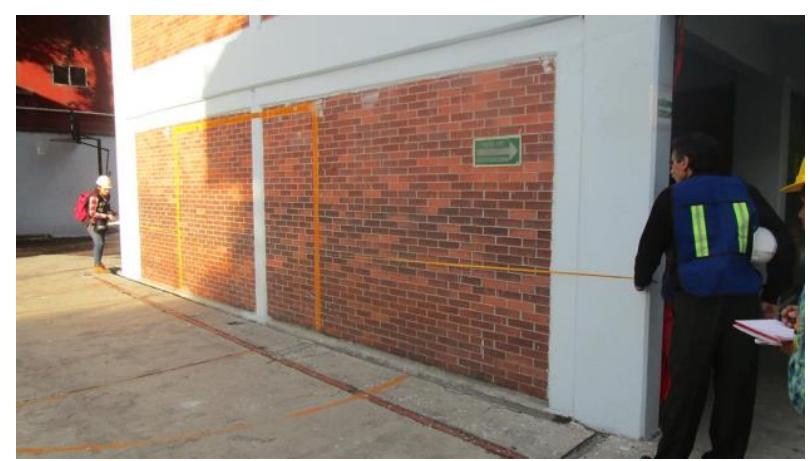

Figura 21. Realización del levantamiento estructural. (DE-DICYG, 2017)

En caso de no contar con información confiable, se recomienda realizar un levantamiento estructural con apoyo de una brigada de topografía que garantice el correcto dimensionamiento de la estructura. Para edificaciones relativamente pequeñas, esta tarea se puede realizar con una brigada reducida que cuente con equipo de medición básico, como son flexómetros, cintas y distanciómetros, etc. Dichos levantamientos deben capturarse en planos o croquis estructurales con ayuda de un software de dibujo, debido a que además de presentarse en los reportes, serán usados para la modelación geométrica de la edificación.

\section{Estudio topográfico}

La información proveniente de este estudio es fundamental para el correcto diagnóstico del comportamiento estructural general de las edificaciones. Para fines de una evaluación, resulta necesaria la verificación de la verticalidad de la construcción y la planimetría en sus niveles, cuyos resultados permitan identificar posibles asentamientos diferenciales entre sus apoyos que puedan ocasionar deformaciones al sistema de piso y a la estructura en general. Aunque estos trabajos son realizados por ingenieros topógrafos, fig. 22, la verificación del cumplimiento de los estados límite reglamentarios asociados al comportamiento de la estructura, estará a cargo del especialista en estructuras o geotecnia.

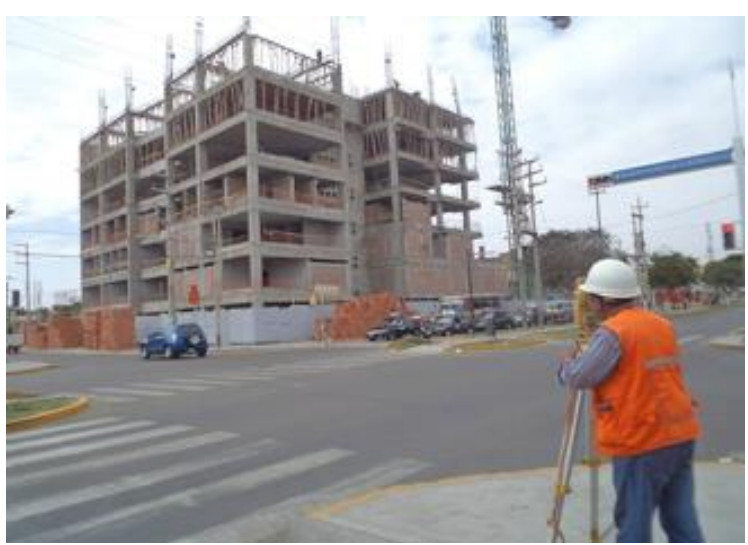

Figura 22. Tareas de la brigada de topografía. (TOPOX ASOCIADOS, 2019) 


\section{Estudios geotécnicos}

La evaluación geotécnica de la cimentación es otra de las etapas fundamentales de la metodología propuesta en este trabajo, ya que las posibles fallas o comportamientos indeseados en el suelo sobre el cual está cimentada la estructura, así como los daños que pudieron ocasionarse en los cimientos producto del movimiento sísmico, también ponen en riesgo la seguridad estructural del inmueble.

El estudio del comportamiento geotécnico deberá realizarse por parte de ingenieros especializados en esta área. Esta actividad abarca desde la inspección de la cimentación y de la zona colindante, hasta la realización de trabajos de campo para la extracción de muestras que permitan determinar las propiedades mecánicas del suelo, necesarias para caracterizar el comportamiento de la cimentación ante las cargas inducidas y verificar el cumplimiento de los estados límite prescritos en la reglamentación vigente aplicable. La cantidad y el tipo de pruebas que se pueden realizar para determinar las características físicas y mecánicas del suelo sobre el cual se encuentra cimentada la edificación, es variada y dependerá del tipo de suelo, cimentación y estructura. Es importante que los resultados de las pruebas sean congruentes y que corroboren los parámetros obtenidos por métodos directos e indirectos.

La justificación para realizar el estudio de mecánica de suelos se basa en el hecho de que las propiedades y características del suelo pueden cambiar a lo largo de la vida de la edificación, o bien, a consecuencia de sismos intensos capaces de dañar la estructura del suelo. Por ello, es importante, caracterizar correctamente el sitio de desplante y, en caso de contar con un estudio de mecánica de suelos al momento de la construcción, verificar los posibles cambios en sus propiedades. El diagnóstico del estado del suelo y de la cimentación quedará a cargo de los ingenieros especialistas en geotecnia; sin embargo, es de importancia que los ingenieros dedicados a la evaluación estructural conozcan las pruebas que deberán realizarse. Los estudios de mecánica de suelos suelen llevarse a cabo mediante exploración directa o indirecta; a su vez, los estudios de exploración directa pueden ser someros o profundos, fig. 23.

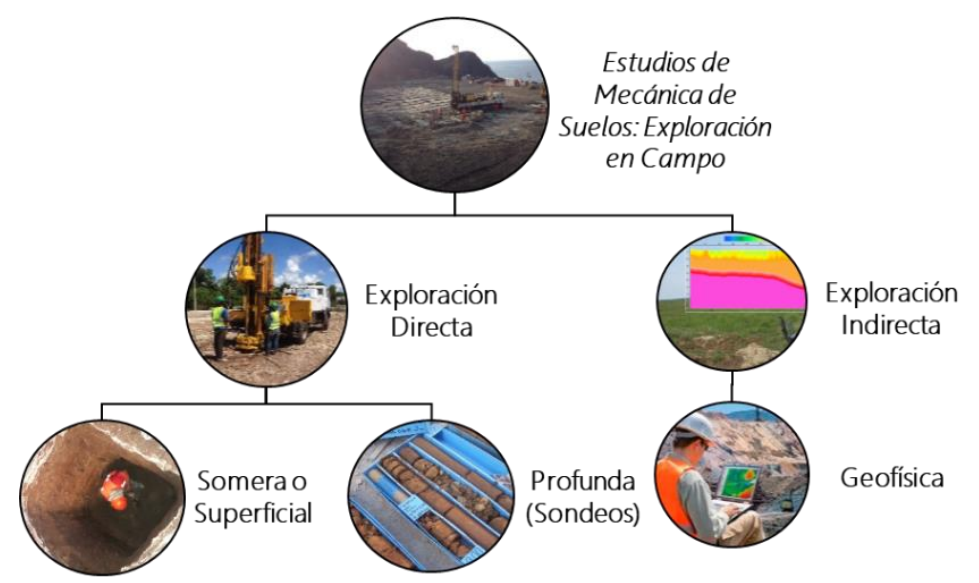

Figura 23. Pruebas de exploración de campo más comunes.

\section{Estudios dinámicos}

La realización de estudios dinámicos en estructuras es, actualmente, una técnica económicamente factible y justificable para estructuras sobre las cuales se tenga duda respecto a su comportamiento ante cargas dinámicas. Este tipo de estudios permiten la determinación de las propiedades que rigen el comportamiento dinámico, a saber: los periodos y modos de vibración, así como, el porcentaje de amortiguamiento crítico. Lo anterior tiene como objetivo evaluar la respuesta ante eventos sísmicos, a partir 
de un modelo estructural representativo de las características y condiciones físicas de la edificación existente. Previo a la obtención de los parámetros dinámicos de una estructura a través de este tipo de estudios, es deseable contar con un modelo preliminar que proporcione información sobre el rango probable en el cual se pueden encontrar dichos valores.

Para realizar un estudio dinámico es necesario llevar a cabo una campaña de medición de vibraciones en campo, en puntos estratégicamente seleccionados dentro de una estructura, cuya localización dependerá del comportamiento dinámico a investigar. Para ello se diseñan una serie de arreglos instrumentales con ayuda de equipos de medición de vibraciones cuya naturaleza y origen pueden ser diversos. Las vibraciones generadas en estructuras suelen clasificarse en dos tipos: vibración forzada y vibraciones ambientales.

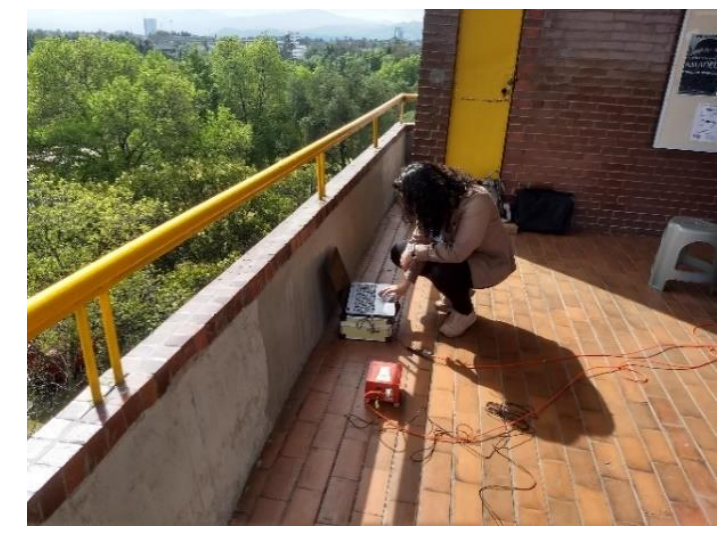

Figura 24. Medición de señales en campo. (DEDICYG, 2019)

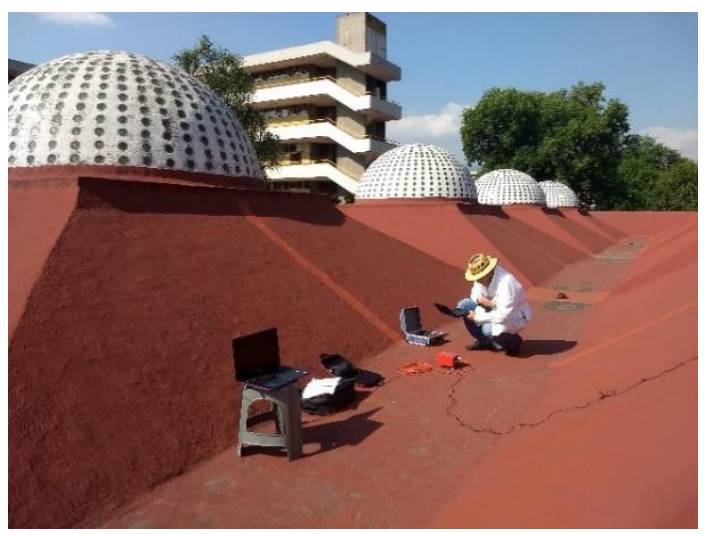

Figura 25. Medición de señales en campo. (DEDICYG, 2019)

Los equipos utilizados comúnmente en estos estudios son acelerómetros o velocímetros, capaces de medir vibraciones en tres direcciones ortogonales (dos traslacionales y una vertical), muchas veces imperceptibles para las personas, figs. 26 y 27; Las señales registradas caracterizan la respuesta del punto de medición ante diversas perturbaciones o excitaciones. Dichas señales son procesadas utilizando diferentes técnicas y métodos numéricos como el análisis de Fourier, el cual permite estudiar las señales en el dominio de la frecuencia, fig. 28. A partir de esta información es posible determinar las frecuencias naturales de vibrar de la estructura y, por ende, sus periodos dominantes de vibrar.

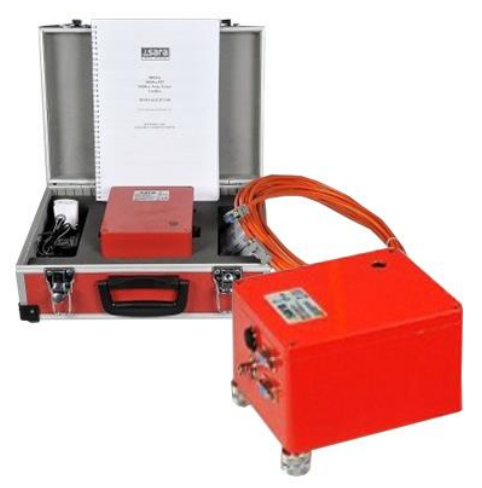

Figura 26. Velocímetro. (DOIT, 2019)

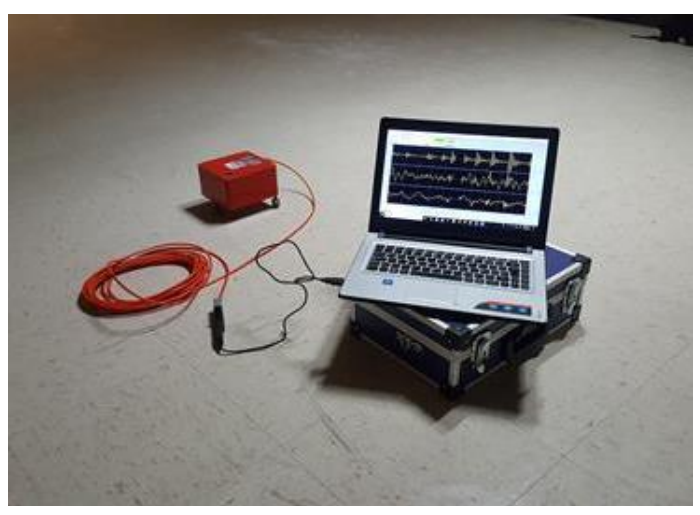

Figura 27. Equipo colocado en campo. (DEDICYG, 2018) 
En la fig. 28 se muestra el panel de control de un programa desarrollado en LabView (Castelán, 2001) con el cual se puede realizar el análisis y procesamiento de señales.

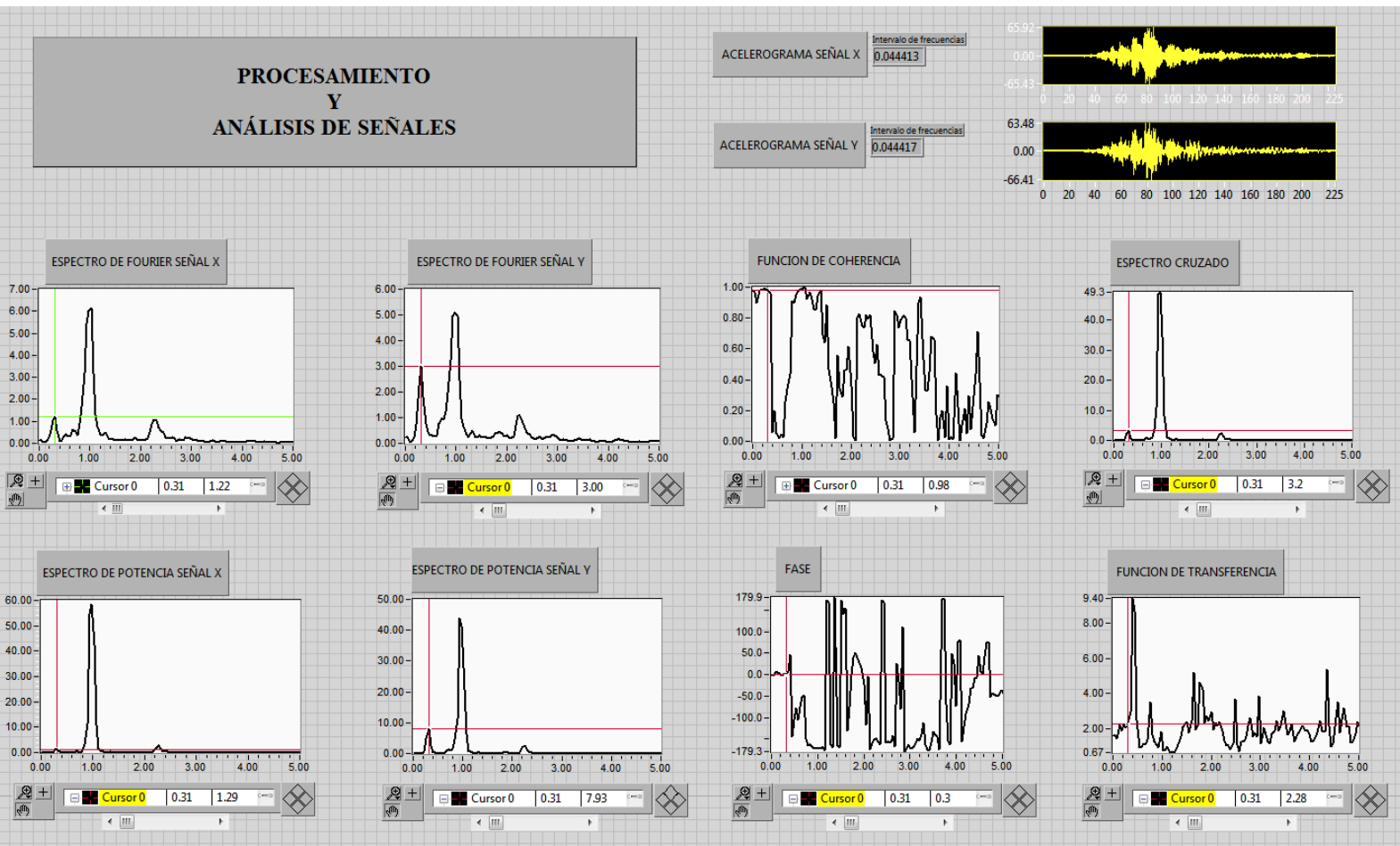

Figura 28. Panel de control del instrumento virtual para la obtención de propiedades dinámicas desarrollado en LabView. (Castelán, 2001)

\section{Vibración forzada}

Los estudios de vibración forzada se caracterizan por generar una perturbación apreciable en la estructura, lo que ocasiona señales con una mayor amplitud y claridad, facilitando la identificación de las propiedades dinámicas de la estructura. Este tipo de excitaciones pueden ser causadas de manera artificial, mediante excitadores mecánicos o dispositivos especiales; y de manera natural, generadas por la ocurrencia de sismos, vientos, oleaje, etc.

\section{Vibraciones ambientales}

Los estudios dinámicos a partir de vibraciones ambientales buscan identificar las frecuencias de vibrar de las edificaciones, a través de excitaciones causadas por diversos agentes ambientales en el interior o en su vecindad, como pueden ser el tránsito de autos o de personas, funcionamiento de equipo y maquinaria, etc.

Debido a que dichas perturbaciones son pequeñas y no se puede controlar su frecuencia ni magnitud, es común que puedan existir niveles de ruido que dificultan el análisis y la interpretación de las señales, por lo cual se requiere contar con una mayor experiencia para realizar este tipo de estudios.

Dada la amplitud de las señales producto de la vibración ambiental, los elementos no estructurales, no desligados en una edificación, pueden contribuir a la rigidez lateral ocasionando variaciones en los 
valores de los periodos. Lo anterior no sucede para otro tipo de vibraciones como las generadas por sismos intensos, donde los elementos no estructurales generalmente se desacoplan o separan de la estructura debido a la severidad del movimiento.

\section{Modelación estructural}

La modelación de la estructura trata una de las etapas más importantes y decisivas de una evaluación, debido a que, a partir de la información recabada, es posible desarrollar un modelo matemático que represente las características geométricas y propiedades mecánicas de los materiales que forman el sistema estructural. Actualmente, la modelación estructural se puede realizar en una variedad muy amplia de programas comerciales y equipos de cómputo de alto rendimiento, por lo que el tiempo requerido para realizar esta tarea es relativamente corto; sin embargo, el personal técnico que la realice debe contar con experiencia en el área y conocimiento pleno de los alcances y limitaciones de estas herramientas.

\section{Calibración del modelo estructural en base a estudios dinámicos}

El proceso de calibración de un modelo estructural con propiedades dinámicas obtenidas a partir de un estudio de vibraciones ambientales o forzadas requiere considerar las condiciones de carga y de rigidez lateral que posee la edificación en su condición actual, es decir, en el momento de realizar la medición de vibraciones. Por ello, durante las etapas de inspección o levantamiento estructural, es importante hacer una estimación de las cargas y/o masas existentes, debido a cargas vivas, peso propio y alguna sobrecarga muerta, que no necesariamente corresponderán a las consideradas en el proyecto original.

Una adecuada estimación de las cargas existentes es fundamental para el cálculo de los periodos y modos de vibrar de la estructura, ya que éstos dependen principalmente de la masa y de su rigidez. Una vez que se ha estimado cuidadosamente la masa, la calibración se enfoca en identificar aspectos estructurales no considerados que afecten la rigidez lateral del edificio, hasta obtener un modelo estructural cuyas propiedades dinámicas obtenidas analíticamente, sean consistentes con las propiedades dinámicas obtenidas experimentalmente.

\section{Estimación de cargas vivas y/o muertas para el desarrollo de modelos preliminares}

Las NTC-CADEE (GCDMX, 2017) establecen tres tipos de cargas en función de su duración e intensidad. Para este apartado, serán de interés las Acciones Permanentes y las Variables. Las primeras, también conocidas como Cargas Muertas son definidas como "...los pesos de todos los elementos constructivos, de los acabados y de todos los elementos que ocupan una posición permanente y tienen un peso que no cambia sustancialmente con el tiempo." (GCDMX, 2017); las segundas llamadas Cargas Vivas se identifican como "...las fuerzas que se producen por el uso y ocupación de las edificaciones y que no tienen carácter permanente” (GCDMX, 2017).

El peso propio de la estructura es comúnmente calculado en la etapa de modelación mediante el uso de software; sin embargo, se recomienda realizar una verificación rápida de la correcta cuantificación del peso de los materiales, con el fin de tener certeza de que el software considera el peso propio real de la estructura. Otro tipo de cargas muertas, usualmente llamadas "sobrecarga muerta", deberán incluir el peso de fachadas, muros divisorios, pretiles, plafones, instalaciones, maquinaria y cargas adicionales que no se encuentren registradas en planos y memorias de cálculo del proyecto original o bien, aquellas generadas por un cambio de uso. 


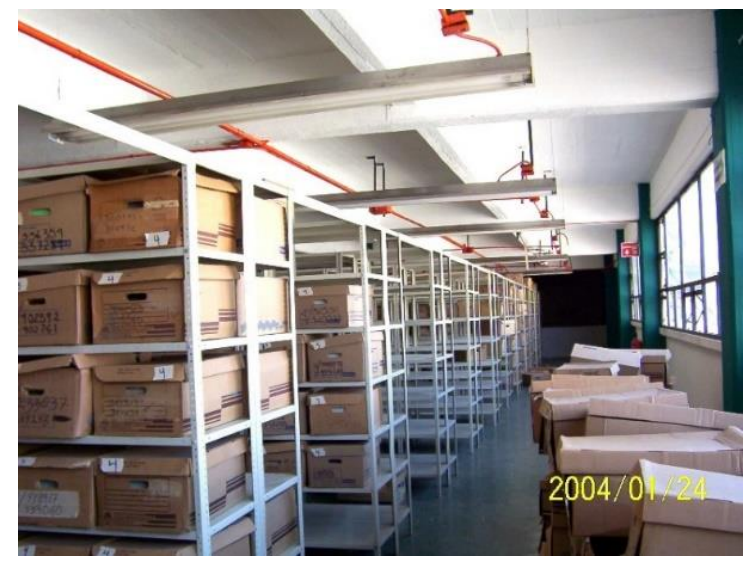

Figura 29. Ejemplo de carga muerta adicional. (DE-DICYG, 2004)

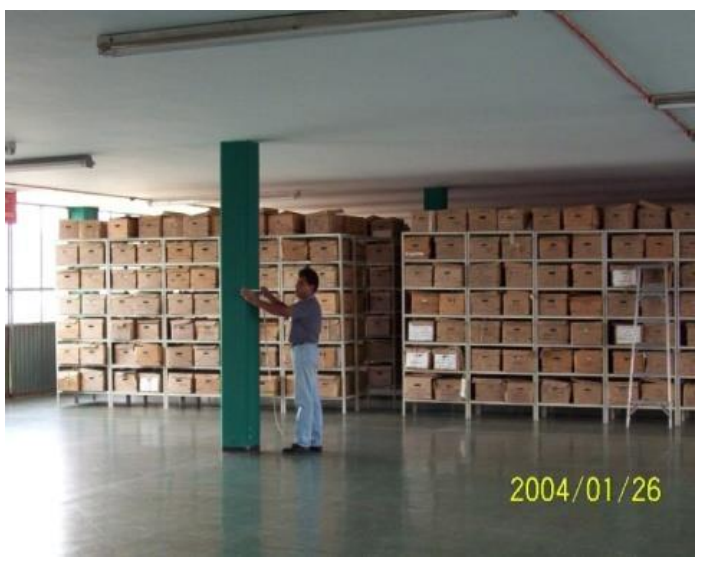

Figura 30. Ejemplo de carga muerta adicional (DE-DICYG, 2004)

Respecto a la carga viva, será necesario hacer una estimación de ésta durante la campaña de mediciones ya que, normalmente las cargas vivas reglamentarias son valores máximos probables, cuyas intensidades no necesariamente se tienen durante este tipo de estudios.

Para el caso de la rigidez, se debe verificar que todos los elementos estructurales estén siendo contemplados correctamente; además se debe verificar si los muros divisorios se encuentran o no correctamente desligados y que los muros de carga hayan sido considerados. Dependiendo de la antigüedad de la construcción, las evidencias de daño detectadas y las pruebas de materiales, entre otros aspectos, será importante considerar un cierto nivel de deterioro en las propiedades geométricas de los elementos estructurales. Al respecto las NTC-DCEC (GCDMX, 2017), recomiendan un deterioro de la inercia en columnas y trabes para fines de evaluación estructural, el cual podrá variar tomando en cuenta lo antes mencionado.

En algunos casos será necesario realizar una visita al inmueble para revisar si existe alguna posible influencia debido a las condiciones de frontera de la estructura, producto de su interacción con otros cuerpos estructurales o construcciones vecinas, es decir, factores en su colindancia que alteren sus frecuencias y formas modales de vibrar.

Una vez tomados en cuenta todos los factores antes descritos, los periodos y formas modales del modelo estructural deben ser sensiblemente similares a los obtenidos mediante estudios dinámicos en campo.

\section{Criterios y acciones de diseño}

Las NTC-CADEE (GCDMX, 2017) establecen los lineamientos para la determinación de las condiciones de carga y sus combinaciones para el diseño de la edificación.

Para realizar una revisión estructural reglamentaria, es necesario colocar en el edificio las cargas muertas y vivas asociadas al nivel de seguridad del reglamento y no las utilizadas para la calibración del modelo. Las cargas muertas estimadas, como peso propio de la estructura, peso de elementos no estructurales, peso de fachada, maquinaria, etc. no cambiarán; sin embargo, deberá sumarse las cargas adicionales que apliquen según las normas. 
En la versión actual de las NTC-DS (GCDMX, 2017), las demandas sísmicas se determinan mediante el Sistema de Acciones Sísmicas de Diseño (SASID), con el que se generan los espectros de diseño sísmico a partir de las coordenadas de la ubicación de la edificación y de algunas características estructurales, definidas a través de diversos factores relativos a la importancia, capacidad dúctil, irregularidad e hiperestaticidad. Para el interior de la República Mexicana, es común que se use el MDOC (CFE, 2017) que cuenta con el software PRODISIS que funciona de manera similar.

Una vez que se han definido todas las acciones a las que puede estar sujeta la estructura, deben establecerse las combinaciones de diseño considerando los efectos bidireccionales establecidos por las NTC-CADEE (GCDMX, 2017) en sus secciones 2.3. y 3.4. En estos apartados también se indican los factores de carga para cada combinación, correspondientes a los diferentes grupos de edificaciones según su importancia.

\section{Análisis estructural}

El análisis estructural tiene como objetivo obtener la respuesta de la estructura a través del cálculo de desplazamientos y fuerzas en los elementos, producto de las diferentes condiciones y combinaciones de carga establecidas por los reglamentos de diseño.

Como se explicó anteriormente, en el caso de estructuras existentes es altamente recomendable que se cuente con un modelo representativo del comportamiento dinámico de la estructura, para evaluar el cumplimiento de los estados límite reglamentarios en sus condiciones reales.

En la actualidad existen numerosas herramientas que realizan este proceso numérico; sin embargo, la calidad de los resultados dependerá del cuidado que se haya tenido en el desarrollo del modelo estructural y de la correcta estimación de las acciones. Otro aspecto importante, es el conocimiento de las hipótesis y limitantes que poseen los programas utilizados para el análisis y diseño, ya que, no deben ser utilizados como "cajas negras".

Es importante que pueda revisarse la consistencia de los resultados mediante algunas pruebas básicas como la comparación de las cargas gravitacionales aplicadas, respecto a las reacciones en la base calculadas por el programa. Para fines de análisis dinámico, empíricamente se ha estimado que el periodo fundamental de vibración $(\mathrm{T})$ de un edificio es aproximadamente igual $0.1 \mathrm{~N}$, donde $\mathrm{N}$ es el número de niveles. De encontrarse resultados inconsistentes del periodo de vibración, es importante revisar los parámetros de los cuales depende, a saber, la masa y la rigidez.

\section{Revisión de estados límite}

Como ya se mencionó, la seguridad estructural estará satisfecha con dos aspectos: Resistencia y Estabilidad. Por ello, el RCDF (GCDMX, 2017) y las NTC (GCDMX, 2017) establecen un conjunto de revisiones que debe cumplir la estructura para considerar que tiene el nivel de seguridad establecido. A continuación, se describen dichas revisiones:

- La primera, denominada revisión del cortante basal mínimo que establece que: "Si en la dirección de análisis se encuentra que la fuerza cortante basal $\mathrm{V}_{\mathrm{o}}$ obtenida con el análisis dinámico modal especificado en la sección 6.1 es menor que $\mathrm{a}_{\min } \mathrm{W}_{\mathrm{o}}$, se incrementarán todas las fuerzas de diseño en una proporción tal que $\mathrm{V}_{\mathrm{o}}$ iguale ese valor; los desplazamientos no se afectarán por esta corrección. $\mathrm{W}_{\mathrm{o}}$ es el peso total de la estructura al nivel del desplante, y $\mathrm{a}_{\min }$ se tomará igual a 0.03 cuando $\mathrm{T}_{\mathrm{s}}<$ $0.5 \mathrm{~s}$ o $0.05 \mathrm{si} \mathrm{T}_{\mathrm{s}} \geq 1.0 \mathrm{~s}$, donde $\mathrm{T}_{\mathrm{s}}$ es el periodo dominante más largo del terreno en el sitio de interés. 
Para valores de $\mathrm{T}_{\mathrm{s}}$ comprendidos entre 0.5 y 1.0 , $\mathrm{a}_{\min }$ se hará variar linealmente entre 0.03 y $0.05 . "$ (GCDMX,2017)

- La segunda, denominada revisión de desplazamientos donde establece que: "La distorsión de entrepiso se define como la diferencia entre los desplazamientos laterales de los pisos consecutivos que lo delimitan dividida entre la diferencia de elevaciones correspondiente. Para efectos de revisión, los desplazamientos laterales se obtienen del análisis realizado con las fuerzas sísmicas de diseño, y deberá considerarse la mayor distorsión de las que se calculan para cada elemento o subsistema vertical contenido en el entrepiso (marcos, muros y cualquier otro elemento vertical)." Y que para efectos de evaluación se verificará el cumplimiento del estado límite de seguridad contra colapso, por lo que "....se revisará que las distorsiones obtenidas con el espectro de diseño definido en el Capítulo 3 , multiplicadas por $\mathrm{Q}_{\mathrm{R}}$, no excedan los valores especificados para la distorsión límite $\left(\gamma_{\max }\right)$ en las tablas 4.2.1, 4.2.2 y 4.2.3, según el sistema estructural que se haya adoptado." (GCDMX, 2017)

- La tercera revisión se refiere al cumplimiento del estado límite de falla de los elementos estructurales, que debe asegurar que "la resistencia de diseño de toda sección con respecto a cada fuerza o momento interno que en ella actúe, sea igual o mayor que el valor de diseño de dicha fuerza o momento interno." (GCDMX, 2017).

- Adicionalmente, se recomienda revisar la inclinación media de la construcción en los casos en los que existan desplomos. Las NTC-DCC (GCDMX, 2017), en su tabla 3.1.1, establecen que la inclinación visible límite de una construcción es igual al porcentaje obtenido de, $100 /\left(100+3 h_{c}\right)$, donde $h_{c}$ es la altura de la construcción en metros.

\section{CONCLUSIONES Y RECOMENDACIONES}

La evaluación integral de la seguridad estructural de una edificación es un proceso que enfrenta diversos obstáculos; sin embargo, la correcta realización de las etapas descritas en este artículo permitirá tener mayor certeza de los resultados obtenidos.

Un aspecto que facilita y ayuda a planear las etapas posteriores, es el contar con información técnica del proyecto original y estudios o trabajos previos, que, en caso de ser confiables, ahorrarán tiempo al evaluador. Por lo anterior, el obtener la mayor cantidad de información útil y confiable, en la etapa inicial, será determinante en la evaluación.

En la mayoría de los casos, los mecanismos de falla, así como los deterioros y daños estructurales en concreto se manifiestan físicamente con fisuras, grietas, corrosión, entre otros; por lo que, el diagnóstico y la documentación de daño son las evidencias de lo que ocurre en la edificación y deberán ser utilizadas para confirmar y validar los efectos estructurales existentes.

Frecuentemente, los factores ambientales, la antigüedad y la falta de mantenimiento, son causantes del deterioro de las propiedades de los materiales y de los elementos, provocando condiciones estructurales diferentes a las originales. Por ello, es de importancia la realización de estudios de calidad de materiales para identificar sus propiedades mecánica para ser capaces de replicar en los modelos las condiciones reales.

Para los casos en los que no se cuenta con información estructural, los levantamientos geométricos y trabajos de campo se convierten en la única fuente de información que identifica el sistema estructural, sus dimensiones y elementos resistentes; por lo que, esta etapa debe realizarse con la mayor exactitud y cuidado posible, evitando errores en las mediciones o pérdidas de información. En los casos en los que se cuente con información estructural, el levantamiento verificará la información y asegurará si existen o no modificaciones a la estructura. 
La estabilidad de una edificación es un aspecto fundamental cuando se habla de seguridad estructural, por lo que, los resultados de los estudios topográficos serán de importancia para la determinación y evaluación del comportamiento general de un sistema en conjunto, permitiendo identificar hundimientos, asentamientos diferenciales y pérdida de verticalidad; así como la identificación de las zonas potenciales de daño en la estructura.

Una vez que el evaluador ha recabado la información correspondiente a las propiedades y estado estructural de la edificación, cuenta con los insumos necesarios para realizar un modelo analítico con ayuda de un software; sin embargo, existe incertidumbre respecto a si el modelo representa a la estructura en condiciones reales. Es por ello, que se considera pertinente la obtención de parámetros dinámicos en sitio producto de un estudio de vibraciones ambientales y su comparación con parámetros dinámicos calculados analíticamente, con lo que es posible desarrollar un modelo representativo de la estructura.

Con base en lo antes expuesto, la evaluación de la seguridad estructural a partir del cumplimiento de los estados límite reglamentarios dependerá, en primera instancia, de la determinación confiable de las acciones a las que estará sometida la estructura a lo largo de su vida útil y, finalmente, de contar con un modelo estructural que represente las condiciones reales de la estructura existente.

Los efectos de los sismos en México y en el mundo, han causado pérdidas económicas y humanas importantes que han intensificado las investigaciones y generado actualizaciones a las normas referentes al diseño sismo resistente. En nuestro país, tales actualizaciones suelen tener un impacto relativamente bajo en la seguridad de la mayoría de las estructuras convencionales existentes, debido principalmente a que la aplicación de estas normas no es retroactiva, excepto en estructuras que hayan sufrido daño o bien las clasificadas como estructuras importantes (Grupo A). Por ello, las deficiencias en la seguridad estructural de un edificio suelen evidenciarse durante o posterior a un evento sísmico intenso.

En zonas de alta sismicidad, como lo son muchas de las regiones de nuestro país, la evaluación y revisión de la seguridad estructural debería realizarse después de un evento sísmico intenso, a diferente nivel, en todas las construcciones y no sólo a las antes mencionadas. La identificación de estructuras vulnerables o aquellas que no cuentan con la seguridad estructural reglamentaria, permitiría tomar medidas preventivas para proteger la integridad de los ocupantes y de las estructuras vecinas. Un censo de este alcance es un problema complejo, social y económicamente; sin embargo, los beneficios serían mayores, si se toman en cuenta los escenarios catastróficos que se han tenido que enfrentar en sismos pasados. Esta medida podría facilitarse si la población fortalece su cultura en cuanto a protección civil.

\section{REFERENCIAS}

@ blogs.20minutos (2016). Fallo por punzonamiento de una piscina. Publicación en el blog Ingeniería en las redes, publicada el 8 de marzo de 2016. Disponible en: http://www.prontubeam.com/Pag_cada_curiosidad.php?Id_curiosidad=32 (Consultado febrero 2019)

@ Mundo_Hormigon (2017). Daños en columnas debido a sismo (efecto de columna corta) Imagen en tweet, publicada el 16 de enero del 2017. Disponible en: https://twitter.com/mundo_hormigon/status/820991680451084288 (Consultado febrero 2019)

ANGULAR 11-18 MX (2017). "19 de septiembre 2017-SISMO 7.1 Richter a las 13:14 Hrs en la Ciudad de México”. Revista en línea ANGULAR 11-18 MX publicada el 21 de septiembre del 2017. Disponible en: http://angular11-18mexico.com.mx/2017/09/21/19-de-septiembre-2017-sismo-7-1-richter-a-las1314-hrs-en-la-ciudad-de-mexico/ (Consultado enero 2019) 
Castelán I. (2001), Determinación Experimental de las Propiedades Dinámicas de un Edificio Reforzado con Disipadores de Energía. Tesis Maestría. Facultad de Ingeniería de la UNAM.

CFE (2015) Manual de Diseño de Obras Civiles, Diseño por Sismo

DE-DICYG (2012, 2017-2019) Archivo fotográfico y documental interno. Departamento de Estructuras de la División de Ingenierías Civil y Geomática de la Facultad de Ingeniería de la UNAM

DOIT. (2019). SRO4, Geobox. Disponible en: http://www.k-doit.com/?tpf=seismometer/site-amplification (Consultado abril 2019)

GCDMX (2017) Normas Técnicas Complementarias. Gaceta Oficial de la Ciudad de México publicada el 15 de diciembre de 2017.

GCDMX (2017) Reglamento de Construcciones para el Distrito Federal. Gaceta Oficial de la Ciudad de México publicada el 15 de diciembre de 2017.

Geofísica Exploraciones (2019). Ingeniera sísmica aplicada a la ingeniería. Blog comercial en línea Geofísica Exploraciones Disponible en http://geofisicaexploraciones.com/ingenieria-sismicaaplicada-a-la-ingenieria/ (Consultado abril 2019)

Geotecniafacil.com (2019). Cálculo Online asientos diferenciales y distorsión angular cimentaciones. Recurso didáctico online Geotecniafacil. Disponible en: http://geotecniafacil.com/asentamientodiferencial-y-distorsion-angular-en-cimentaciones/ (Consultado febrero 2019).

Hilti México (2019). Sistema Ferroscan PS 250 - Escáneres de concreto Disponible en: https://www.hilti.com.mx/c/CLS_MEA_TOOL_INSERT_7127/CLS_CONCRETE_SCANNERS_7 $\underline{127 / r 41791}$ (Consultado Marzo 2019)

IDC (2019). Asentamientos en suelos. Blog en línea Disponible en: https://ingeniero-decaminos.com/asentamientos-en-suelos/ (Consultado febrero 2019)

INDECAL (2019). Extracción de Núcleos de Concreto Disponible en: https://www.indecalsrl.com/proyecto/extraccion-nucleos-de-concreto/ (Consultado marzo 2019)

Materiales Ramonsón (2013). La carbonatación en el concreto reforzado. Blog en línea publicado el 28 de junio del 2013. Disponible en: https://materiales-ramonson.blogspot.com/2013/06/la-carbonatacionen-el-concreto.html (Consultado febrero 2019)

Protocolo (2015). Los sismos y su efecto en las estructuras. Revista en línea Protocolo publicada el 18 de septiembre del 2015. Disponible en: http://www.protocolo.com.mx/internacional/los-sismos-y-suefecto-en-las-estructuras/ (Consultado enero 2019)

TOPOX ASOCIADOS S.A.C. (2019). Equipos Topográficos, Especialistas en servicios de topografía profesional. Blog comercial en línea TOPOX, Disponible en: https://www.topoxperu.com/experiencia 